\title{
Atividade física em mulheres brasileiras
}

\section{Pbysical activity in brazilian women}

A atividade física (AF) é definida como gasto energético por meio de movimentos corporais produzidos pela musculatura esquelética ${ }^{1}$. Contempla atividades em diversos contextos, como lazer, meio de transporte, tarefas domésticas e trabalho ${ }^{2}$. Dentre as atividades importantes para manutenção do equilíbrio, agilidade e capacidade funcional, as mais utilizadas são as caminhadas, exercícios de força e alongamentos. Os exercícios físicos regulares podem prevenir obesidade, diabetes, dislipidemia e doenças cardíacas ${ }^{3}$. Sabe-se que mulheres têm menor AF em relação aos homens, principalmente em atividade de lazer; porém, participam mais do que os homens em atividades caseiras ${ }^{4}$. A AF pode reduzir o risco de diferentes doenças por melhorar o metabolismo e aumentar a massa muscular, e a sua promoção tem sido objeto de campanhas públicas no Brasil e em muitos outros países ${ }^{5}$. A Associação Médica Brasileira (AMB), o Conselho Federal de Medicina (CFM) e a Sociedade Brasileira de Medicina do Esporte (SBME) recomendam aos profissionais de saúde que estimulem a prática de atividades físicas regulares com o objetivo de diminuir o risco de doenças 6 .

Um estudo no qual se investigou o efeito do nível de AF sobre a morbidade de mortalidade feminina concluiu que mulheres que realizavam atividades recreativas enquanto jovens mantiveram alto nível de atividade física posteriormente e apresentavam menor mortalidade ${ }^{7}$. Atividade física, além dos benefícios físicos, tem um papel importante na qualidade de vida, que engloba domínios, como capacidade funcional, estado emocional, interação social, atividade intelectual, autocuidado, suporte familiar, estado de saúde, valores culturais, éticos, religiosos, estilo de vida, satisfação com o emprego e/ou com atividades diárias no ambiente em que se vive. Está, também, relacionada à autoestima e ao bem estar individual ${ }^{8}$. Observamos em Campinas (SP) que mulheres que praticavam $\mathrm{AF}$ regularmente apresentaram melhores habilidades sensoriais, melhor autopercepção de saúde e menor uso de medicamentos; porém, despenderam muito tempo de suas vidas em atividades na posição sentada, para assistir televisão, locomoverem-se nos automóveis, ouvir rádio e receber visitas ${ }^{9}$. Considerando as recomendações atuais de se praticar pelo menos 30 min diários de AF com intensidade moderada ou rigorosa para se conseguir os benefícios em relação à saúde ${ }^{10}$, essa população está aquém de obter os benefícios que podem advir com a prática de AF regulares, pois atividades realizadas na posição sentada são consideradas de baixo gasto energético e associam-se ao sedentarismo, ao risco de obesidade e suas consequências, como doenças cardiovasculares e dependência física ${ }^{11}$. Nessa mesma população de

Correspondência Aarõo Mendes Pinto Neto Departamento de Tocoginecologia da Faculdade de Ciências Médicas da Universidade Estadual de Campinas - UNICAMP Rua Alexander Fleming, 101 - Cidade Universitárí "Zeferino Vaz" - Barão Geraldo (EP: 13083-881 Campinas (SP), Brasil Recebido $21 / 09 / 2012$
Departamento de Tocoginecologia da Faculdade de Ciências Médicas da Universidade Estadual de Campinas - UNICAMP Campinas (SP), Brasil.

'Departamento de Tocoginecologia da Faculdade de Ciências Médicas da Universidade Estadual de Campinas - UNICAMP Campinas (SP), Brasil.

2Programa de Pós-Doutorado do Departamento de Tocoginecologia da Faculdade de Ciências Médicas, Universidade Estadual de Campinas - UNICAMP - Campinas (SP), Brasil.

${ }^{3}$ Departamento de Tocoginecologia da Faculdade de Ciências Médicas, Universidade Estadual de Campinas - UNICAMP Campinas (SP), Brasil. 
mulheres residentes em Campinas, com 60 ou mais anos de idade, constatamos que o tempo despendido com AF, como lazer, foi a menor em relação a outros tipos de atividade física, possivelmente por condição social menos favorecida (aproximadamente $60 \%$ delas pertenciam a extrato social C e D). Nesse grupo, as mulheres que relataram caminhadas regulares como meio de locomoção, a AF como transporte associou-se a melhor qualidade de vida ${ }^{12}$. Investigações têm mostrado que indivíduos que praticam AF como meio de transporte são fisicamente ativos e consideram fáceis atividades como fazer compras, visitar amigos/parentes e frequentar centros de lazer. No entanto, segundo Hallal et al. ${ }^{13}$ o lazer é o domínio mais frequentemente investigado no Brasil e em outros países. Estudo por inquérito telefônico no qual se avaliou a AF no lazer, na população brasileira, registrou que somente $12 \%$ das mulheres com 45 anos ou mais foram ativas no lazer, percentual esse menor do que o relatado na população masculina. Nesse inquérito, a caminhada foi a modalidade mais comum de AF de lazer para ambos os sexos, associou-se diretamente com a maior escolaridade, e indiretamente com o envelhecimento ${ }^{14}$. Apesar dos níveis de AF ocupacionais serem baixos, observa-se crescente interesse pela implementação de AF realizadas no trabalho e no seu deslocamento e nas atividades domésticas, por fazerem parte do cotidiano das pessoas e parecerem mais viáveis de serem realizadas ${ }^{13}$. Em relação às tendências de $\mathrm{AF}$, na cidade de Pelotas $(\mathrm{RS})^{15}$, observou-se diminuição de indivíduos ativos entre 2002 e 2007. Por outro lado, no estado de São Paulo $(\mathrm{SP})^{16}$, relatou-se aumento dos níveis de AF total. No entanto, estudos que relatem tendências de AF são escassos.

Cabe destacar que a consolidação do Sistema de Vigilância de Fatores de Risco e Proteção para Doenças Crônicas por Inquérito Telefônico (VIGITEL), do Ministério da Saúde, permitiu que dados nacionais sobre a prática de atividade física em adultos de 18 anos ou mais estejam disponíveis ${ }^{17}$. O sistema foi implementado em 2006 nas 26 capitais brasileiras e no Distrito Federal e foi coordenado pelo Ministério da Saúde, por intermédio da Secretaria de Vigilância em Saúde, com o apoio da Secretaria de Gestão Estratégica e Participativa e do Núcleo de Pesquisas Epidemiológicas em Nutrição e Saúde (NUPENS) da Universidade de São Paulo. O VIGITEL tem contado também com o apoio do grupo técnico do Behavioral Risk Factor Surveillance System (BRFSS) do Centro de Controle de Doenças (CDC), dos Estados Unidos. Por meio de entrevistas telefônicas assistidas por computador, o VIGITEL utiliza-se de amostras probabilísticas da população adulta de 18 anos ou mais, residente em domicílios servidos por pelo menos uma linha telefônica fixa no ano da pesquisa, nas 26 capitais dos estados brasileiros e no Distrito Federal, totalizando mais de 54.000 entrevistas ao ano ${ }^{18}$.

Os dados do VIGITEL permitiram conhecer a evolução da frequência da inatividade física de 2009 a 2011.
Em 2009, 15,3\% das mulheres eram inativas sendo que, em 2011, 13,9\% delas foram categorizadas como inativas fisicamente ${ }^{19}$. Os objetivos do VIGITEL, além de medir a prevalência de fatores de risco e proteção para doenças não transmissíveis na população brasileira, é subsidiar ações de promoção da saúde e da prevenção de doenças. Nesse sentido, a prática de AF regular deve ser sempre lembrada e recomendada por todos os profissionais da saúde a seus clientes, independentemente de idade, gênero e estado social.

Diversos fatores sociodemográficos podem influenciar o comportamento da prática da AF em grupos populacionais ${ }^{20}$. O nível socioeconômico, a escolarização, a idade, a etnia, entre outras variáveis, vêm sendo relatados como determinantes da $\mathrm{AF}$ em seus diferentes domínios. Por outro lado, apesar da existência de poucos estudos que abordem a associação entre percepção de violência/policiamento e AF, sugere-se que a percepção da violência está associada a medo e AF, ou seja, o ambiente mais seguro aumenta a possibilidade de prática de AF. O fato é que os níveis de AF na população brasileira estão relativamente estáveis no tempo livre e nas atividades domésticas entre 2006 e 2009, mas aumentaram no deslocamento e nas atividades ocupacionais, acarretando numa diminuição no percentual de inativos no país.O comportamento sedentário, expresso pelo tempo despendido assistindo televisão, apresentou leve decréscimo em 2008 e 2009 se comparado a 2006 e $2007^{19}$.

Atualmente, cerca de 1000 entes federados recebem financiamento do Ministério da Saúde para o desenvolvimento de programas de promoção da $\mathrm{AF}^{21}$, além de outros programas locais implantados há longo tempo, como Curitiba Ativa, Agita São Paulo, Academia da Cidade de Recife, Serviço de Orientação do Exercício. Os únicos dados de tendências temporais 'naturais' de AF disponíveis são os coletados em Pelotas, uma cidade de porte médio no sul do país, no qual, porém, não há qualquer intervenção populacional para promoção da AF. Embora a capacidade de generalização de tais informações para todo o país seja bastante limitada, os dados de Pelotas sugerem diminuição nos níveis de AF com o passar dos anos, diferentemente do observado no VIGITEL. Portanto, o fato dos dados do VIGITEL mostrarem estabilização, ou até mesmo aumento nos níveis de AF (deslocamento), sugere um possível impacto da rede nacional de promoção da saúde. De acordo com esses mesmos autores, ao longo dos últimos anos o Ministério da Saúde tem estabelecido com os entes federados, no pacto pela vida, a inclusão de indicadores de elevação dos níveis de AF com os municípios.

Também não podemos esquecer que o envelhecimento da população brasileira é um fato real, especialmente entre as mulheres. Nesse sentido, a prevenção de doenças crônicas não transmissíveis deve ser um objetivo claro no atendimento e nas políticas públicas de atenção à saúde. 
Não existem dúvidas que a AF regular desempenha um papel de destaque no bem-estar geral. Recente revisão sistemática ${ }^{22}$ destacou o papel da atividade física também como uma intervenção efetiva para a prevenção de quedas e suas consequências em idosos de ambos os sexos que vivem na comunidade. Destacamos a importância dos dados gerados pelo VIGITEL como indicadores claros de ações que visem à minimização dos riscos para doenças crônicas não transmissíveis no Brasil, e como uma diretriz fundamental para a promoção da saúde e melhoria da atenção à população brasileira. Contempla, além da AF, a prevalência de tabagismo, consumo alimentar, excesso de peso e obesidade, consumo de bebidas alcoólicas, prevenção de câncer, indicadores importantes de saúde e bem-estar ${ }^{19}$.
Sabendo-se que o sedentarismo é um fator de risco modificável levando à prevenção das doenças crônicas não transmissíveis e para a melhoria da qualidade de vida, enfatiza-se a importância da conscientização das pacientes a esse respeito e do incentivo à AF. Em inúmeras circunstâncias o ginecologista atua como clínico da mulher e na maioria das vezes é o único médico que ela procura e ouve. Portanto, podemos ter papel importante na modificação do estilo de vida das nossas pacientes, mostrando que as alternativas disponíveis para a prática da AF muitas vezes estão ao alcance das pessoas, mas passam despercebidas. No entanto, para que essa prática da AF torne-se mais frequente, faz-se necessária a presença de locais públicos apropriados e seguros.

\section{Referências}

1. Sims J, Hill K, Hunt S, Haralambous B. Physical activity recommendations for older Australians. Australas J Ageing. 2010;29(2):81-7.

2. Annear M, Cushman G, Gidlow B. Leisure time physical activity differences among older adults from diverse socioeconomic neighborhoods. Health Place. 2009; 15(2):482-90.

3. Franchi KMB, Montenegro Junior RM. Atividade física: uma necessidade para a boa saúde na terceira idade. Rev Bras Promoç Saúde. $2005 ; 18(3): 152-6$.

4. Jurj AL, Wen W, Gao YT, Matthews CE, Yang G, Li HL, et al. Patterns and correlates of physical activity: a cross-sectional study in urban Chinese women. BMC Public Health. 2007;7:213.

5. Jurakic D, Pedisic Z, Greblo Z. Physical activity in different domains and health-related quality of life: a population-based study. Qual Life Res. 2010;19(9): 1303-9.

6. Silva RB, Costa-Paiva L, Pinto Neto AM, Braga AA, Morais SS. Atividade física habitual e risco cardiovascular na pós-menopausa. Rev Assoc Med Bras. 2006;52(4):242-6.

7. Nitz JC, Chay NL. Changes in activity level in women aged 40-80 years. Climacteric. 2007;10(5):408-15.

8. Dalla Vecchia R, Ruiz T, Bocchi SCM, Corrente JE. Qualidade de vida na terceira idade: um conceito subjetivo. Rev Bras Epidemiol. 2005;8(3):246-52.

9. Carvalho ED, Valadares ALR, Costa-Paiva LH, Pedro AO, Morais SS, Pinto-Neto AM. Physical activity and quality of life in women aged 60 or older: associated factors. Rev Bras Ginecol Obstet. 2010;32(9):433-40.

10. White SM, Wójcicki TR, McAuley E. Physical activity and quality of life in community dwelling older adults. Health Qual Life Outcomes. $2009 ; 7: 10$.

11. Stewart KJ. Physical activity and aging. Ann N Y Acad Sci. 2005;1055:193-206

12. Valadares ALR, Carvalho ED, Costa-Paiva LH, Morais SS, Pinto-Neto AM. Association between different types of physical activities and quality of life in women aged 60 years or over. Rev Assoc Med Bras. 201 1;57(4):450-5.

13. Hallal PC, Dumith SC, Bastos JP, Reichert FF, Siqueira FV, Azevedo MR. Evolução da pesquisa epidemiológica em atividade física no Brasil: revisão sistemática. Rev Saúde Pública. 2007;41(3):453-60.

14. Knuth AG, Bacchieri G, Victora CG, Hallal PC. Changes in physical activity among Brazilian adults over a 5-year period. J Epidemiol Community Health. 2010;64(7):591-5

15. Matsudo VK, Matsudo SM, Araújo TL, Andrade DR, Oliveira LC, Hallal PC. Time trends in physical activity in the state of São Paulo, Brazil: 2002-2008. Med Sci Sports Exerc. 2010;42(12):2231-6.

16. Brasil. Ministério da Saúde. Secretaria de Vigilância em Saúde. Secretaria de Gestão Estratégica e Participativa. VIGITEL Brasil 2008: vigilância de fatores de risco e proteção para doenças crônicas por inquérito telefônico. Brasília (DF): Ministério da Saúde; 2009.

17. Malta DC, Moura EC, Castro AM, Cruz DKA, Morais Neto OL, Monteiro CA. Padrão de atividade física em adultos brasileiros: resultados de um inquérito por entrevistas telefônicas, 2006. Epidemiol Serv Saúde. 2009;18(1):7-16.

18. Silva Jr JB. Inquéritos nacionais de saúde. Rev Bras Epidemiol. 2011;14 Supl 1:3-4

19. Brasil. Ministério da Saúde. Secretaria de Vigilância em Saúde. Secretaria de Gestão Estratégica e Participativa. VIGITEL Brasil 2011 : vigilância de fatores de risco e proteção para doenças crônicas por inquérito telefônico. Brasília (DF): Ministério da Saúde; 2012.

20. Pitanga FJG, Lessa I, Barbosa PJB, Barbosa SJO, Costa MC, Lopes AS. Fatores sociodemográficos associados aos diferentes domínios da atividade física em adultos de etnia negra. Rev Bras Epidemiol. 2012;15(2):363-75.

21. Hallal PC, Knuth AG, Reis RS, Rombaldi AJ, Malta DC, Iser BPM, Bernal RTI, Florindo AA. Time trends of physical activity in Brazil (2006-2009). Rev Bras Epidemiol. $2011 ; 14(3): 53-60$.

22. Gillespie LD, Robertson MC, Gillespie WJ, Lamb SE, Gates S, Cumming RG, et al. Interventions for preventing falls in older people living in the community. Cochrane Database Syst Rev. 2009;(2):CD007146. 